Original paper

\title{
The usefulness of commercially available serological tests in the diagnosis and monitoring of treatment in patients with alveolar echinococcosis
}

\author{
Małgorzata Sulima ${ }^{1}$, Beata Szostakowska², Wacław Nahorski ${ }^{1,3}$, Katarzyna Sikorska ${ }^{1,4}$, Wojciech Wołyniec ${ }^{5}$ Piotr Wąiz \\ 'Department of Tropical and Parasitic Diseases, University Centre of Maritime and Tropical Medicine (UCMTM) in Gdynia, Medical University \\ of Gdansk, Poland \\ ${ }^{2}$ Department of Tropical Parasitology, Chair of Tropical Medicine and Parasitology, Institute of Maritime and Tropical Medicine, Faculty \\ of Health Sciences, Medical University of Gdansk, Poland \\ ${ }^{3}$ Department of Tropical and Parasitic Diseases, Institute of Maritime and Tropical Medicine, Faculty of Health Sciences, Medical University \\ of Gdansk, Poland \\ ${ }^{4}$ Department of Tropical Medicine and Epidemiology, Institute of Maritime and Tropical Medicine, Faculty of Health Sciences, Medical \\ University of Gdansk, Poland \\ ${ }^{5}$ Department of Occupational, Metabolic and Internal Medicine, Institute of Maritime and Tropical Medicine, Faculty of Health Sciences, \\ Medical University of Gdansk, Poland \\ ${ }^{6}$ Department of Nuclear Medicine, Medical University of Gdansk, Poland
}

\begin{abstract}
Aim of the study: To assess the clinical usefulness of serological tests in the diagnosis and monitoring of treatment of patients with alveolar echinococcosis (AE).

Material and methods: The results of serological tests, i.e. Echinococcus multilocularis ELISA (Bordier Affinity Products) and Echinococcus Western Blot IgG (LDBIO Diagnostic), of 66 patients were analysed. Duration of follow-up was two years after diagnosis. In the second phase of the study 11 sera obtained from the patients undergoing surgical treatment, in whom the results of Echinococcus Western Blot lgG assay were still positive, were additionally tested with Anti-Echinococcus EUROLINE-WB (IgG) assay.

Results: Statistically significant negativization of the Echinococcus multilocularis ELISA test was observed in the group of patients who underwent radical surgery or liver transplantation. Negativization of Echinococcus Western Blot lgG assay results was observed in some patients, among both those who received conservative treatment and those who underwent surgery, but no statistically significant differences were found between treatment groups. In 54.5\% of cases the Anti-Echinococcus EUROLINE-WB (IgG) test result was negative when the results of the Echinococcus Western Blot lgG assay were still positive.

Conclusions: Echinococcus multilocularis ELISA proved to be useful in assessing the activity of AE in a group of patients who underwent radical surgery or liver transplantation. The results of our study suggest that Anti-Echinococcus EUROLINE-WB (IgG) is a more dynamic test, which at the time of disappearance of AE activity becomes negative earlier.
\end{abstract}

Key words: serology, echinococcosis, alveolar echinococcosis.

Address for correspondence

Małgorzata Sulima, Department of Tropical and Parasitic Diseases, University Centre of Maritime and Tropical Medicine (UCMTM) in Gdynia, Medical University of Gdansk, 9 B Powstania Styczniowego St., 81-519 Gdynia, Poland,

e-mail: m.sulima@poczta.fm 


\section{Introduction}

Echinococcosis is a parasitic disease caused by tapeworms of the genus Echinococcus. The two most common forms of this disease, caused by different tapeworm species, are cystic echinococcosis (CE) and alveolar echinococcosis (AE). CE and AE present different lesion patterns in imaging examinations and are characterized by different clinical course, and thus require different therapeutic approaches. Since the early 1920s, a significant increase in the incidence of AE in Central and Eastern Europe, including Poland [1], has been noted.

Alveolar echinococcosis is caused by the larval form of the tapeworm Echinococcus multilocularis [2]. The most frequent site of primary parasitic lesions is the liver [3]. Clinically, the parasitic invasion is characterized by a long-lasting asymptomatic phase. Often, at the onset of symptoms, imaging examination reveals the presence of a large tumour-like mass in the liver suggesting a proliferative process $[4,5]$. In some cases, at the time of diagnosis, extrahepatic lesions are also visible due to spread by continuity to adjacent structures or metastasizing via the haematogenic route to distant organs such as the lung or central nervous system (CNS). Patients in such an advanced stage of the disease are deprived of the opportunity of optimal treatment, which is radical surgery combined with temporary pharmacological treatment. Patients who are not eligible for surgery require treatment with benzimidazoles for many years, sometimes for the rest of their lives. In some cases, liver transplantation (LTx) remains the only chance to save the patient's life.

Serological blood tests are, besides imaging studies, as well as histopathological and molecular examinations, important tools in the diagnosis of AE. They are also useful in monitoring the effectiveness of treatment and in follow-up after the termination of pharmacotherapy, due to the risk of recurrence, especially in the group of patients receiving immunosuppression. These tests also proved to be useful in screening examinations carried out in the areas of increased incidence of the disease observed due to the increased number of final hosts $[6,7]$. Serological diagnosis is usually based on commercially available immunoenzymatic (ELISA) and immunoblot assays containing recombinant and purified Echinococcus antigens. Immunochromatographic tests are mainly useful as a screening tool in endemic areas.

The study analysed the results of serological tests performed at diagnosis and during treatment of $\mathrm{AE}$ in patients hospitalized at the University Centre of Maritime and Tropical Medicine (UCMTM) between 2000 and 2016. During this period, the screening test used by the UCMTM was Echinococcus granulosus ELISA
(Bordier Affinity Products SA, Crissier, Switzerland) for detecting the presence of IgG antibodies against larval Echinococcus antigens. To confirm the diagnosis and differentiate between $\mathrm{CE}$ and $\mathrm{AE}$, Echinococcus Western Blot IgG (LDBIO Diagnostics, Lyon, France) and Echinococcus multilocularis ELISA (Bordier Affinity Products SA, Crissier, Switzerland) detecting Em2plus antigen were used. In 2016, serological diagnostics was extended to include the commercially available tests Anti-Echinococcus EUROLINE-WB (IgG) and EUROIMMUN Anti-Echinococcus ELISA (IgG), EUROIMMUN US Inc. Medical Diagnostics. Simultaneous use of various serological tests increases the chance of establishing the correct diagnosis of AE, thus avoiding invasive diagnostic methods, such as liver biopsy or exploratory laparotomy. Such an approach is also helpful in assessing the effectiveness of treatment, including determination of the moment when pharmacological treatment can be safely withdrawn. Benzimidazole therapy is associated with potential side effects, including toxic liver damage or leukopenia, and in Poland additionally imposes a large financial burden on the patient.

The aim of the study was to assess the clinical usefulness of commercially available serological tests, Echinococcus multilocularis ELISA and the immunoblot Echinococcus Western Blot IgG, in the diagnosis and monitoring of treatment of patients with $\mathrm{AE}$, based on the analysis of data from the follow-up of patients treated at the Department of Tropical Diseases and Parasitology, UCMTM. Also, the study aimed to determine whether extended diagnostics including the Anti-Echinococcus EUROLINE-WB (IgG) immunoblot assay, performed at the time of Echinococcus multilocularis ELISA negativization in the group of patients undergoing surgical treatment in whom the result of Echinococcus Western Blot IgG assay is still positive, enables a more accurate assessment of parasitic disease activity.

\section{Material and methods}

The analysis included the results of serological tests, i.e. Echinococcus multilocularis ELISA (Bordier Affinity Products SA) and Echinococcus Western Blot IgG (LDBIO Diagnostics), performed at the time of diagnosis and during the treatment of 66 patients hospitalized in the Department of Tropical Diseases and Parasitology, UCMTM between 2000 and 2016. Study participants were patients with probable and certain diagnosis of AE. According to the WHO-Informal Working Group on Echinococcosis (WHO-IWGE), the probable diagnosis was based on the positive re- 
sults of serological tests and a typical pattern of lesions in imaging examinations. The diagnosis was considered as certain in patients in whom the disease was additionally confirmed by histopathological or molecular (PCR) examination. Serological tests were performed in the Department of Tropical Parasitology, Institute of Maritime and Tropical Medicine according to the manufacturers' instructions. The result of the Echinococcus multilocularis ELISA test was considered as negative when the absorbance of the analysed sample was lower than the absorbance of the weak positive (cut-off) serum. The result was positive when the absorbance of the analysed sample was higher than the absorbance of the weak positive serum (IgG antibody concentration against Echinococcus multilocularis Em2-Em18 antigens was considered as clinically significant). The Echinococcus Western Blot IgG assay is based on antigen extracts derived from the E. multilocularis larval form. Bands at 7, 12, 15, 24, and 26 to $28 \mathrm{kDa}$ are common to both Echinococcus species. However, their intensity and arrangement in most cases allow for differentiation between $\mathrm{CE}$ and AE. There are five band patterns that can be obtained in immunoblot assay - P1 to P5. Patterns P1 (isolated $7 \mathrm{kDa}$ band only) and P2 (7 kDa band + large diffuse $17 \mathrm{kDa}$ band) are indicative of CE diagnosis. Patterns P3 (26-28 band + the narrow 16 and/or $18 \mathrm{kDa}$ bands) confirm the diagnosis of AE. Patterns P4 (isolated 26-28 kDa band only) and P5 (association of the $7+26-28 \mathrm{kDa}$ bands) do not allow for differentiation between $\mathrm{CE}$ and AE. The patients $(n=66)$ were assigned to one of four groups depending on the method of treatment used: receiving pharmacological treatment $(n=25)$, radical surgery $(n=21)$, non-radical surgery $(n=10)$ and liver transplantation (LTx) $(n=10)$. In all the patients both tests were performed at diagnosis. During the treatment, serum samples of all patients were assessed using Echinococcus multilocularis ELISA. Echinococcus Western Blot IgG test was performed in 47 patients (71.2\%). Due to the fact that in some patients after surgery or LTx, at the moment of negativization of the Echinococcus multilocularis ELISA test, the results of the Echinococcus Western Blot IgG assay remained positive, 11 sera obtained from such 11 patients were used in the second phase of the study. Five samples were from patients after radical surgery, two from patients who underwent non-radical surgery and four from LTx patients. The sera were stored under freezing conditions at $-60^{\circ} \mathrm{C}$. These 11 sera samples underwent additional testing with Anti-Echinococcus EUROLINE-WB (IgG) assay, performed in accordance with the manufacturer's instructions. The results were read using EUROLineScan software.

\section{Results}

The Echinococcus multilocularis ELISA test result was positive in all patients at the time of diagnosis. During the two-year follow-up, statistically significant negativization of the results of this test was observed in the group of patients who underwent radical surgery or LTx (Table 1). The negativization occurred

Table 1. Number of subjects ( $M$ ) and the percentage values calculated against the sum specified for the given rows (NR) and columns (NC). CTr - patients receiving pharmacological treatment; NRS - patients who underwent non-radical surgery; RS - patients who underwent radical surgery; LTx - patients after liver transplantation. Fisher's exact test for count data was used to examine the independence between the features described in the columns and rows. $P$-value $=0.0000000001392$

\begin{tabular}{|c|c|c|c|c|}
\hline Patients & & $\begin{array}{l}\text { Elisa Em2plus test results negative } \\
\text { in the course of two-year treatment }\end{array}$ & $\begin{array}{l}\text { Elisa Em2plus test results positive } \\
\text { in the course of two-year treatment }\end{array}$ & $\begin{array}{l}\text { All (Elisa Em2plus test positive } \\
\text { at the time of diagnosis) }\end{array}$ \\
\hline \multirow[t]{3}{*}{$\mathrm{CTr}$} & $N$ & 0 & 25 & 25 \\
\hline & NR & $0 \%$ & $100 \%$ & $100 \%$ \\
\hline & NC & $0 \%$ & $64.1 \%$ & $37.9 \%$ \\
\hline \multirow[t]{3}{*}{ NRS } & $N$ & 2 & 8 & 10 \\
\hline & NR & $20 \%$ & $80 \%$ & $100 \%$ \\
\hline & NC & $7.4 \%$ & $20.5 \%$ & $15.2 \%$ \\
\hline \multirow[t]{3}{*}{ RS } & $N$ & 17 & 4 & 21 \\
\hline & NR & $81 \%$ & $19 \%$ & $100 \%$ \\
\hline & NC & $63 \%$ & $10.3 \%$ & $31.8 \%$ \\
\hline \multirow[t]{3}{*}{ LTx } & $N$ & 8 & 2 & 10 \\
\hline & NR & $80 \%$ & $20 \%$ & $100 \%$ \\
\hline & NC & $29.6 \%$ & $5.1 \%$ & $15.2 \%$ \\
\hline \multirow[t]{3}{*}{ Total } & $N$ & 27 & 39 & 66 \\
\hline & NR & $40.9 \%$ & $59.1 \%$ & $100 \%$ \\
\hline & NC & $100 \%$ & $100 \%$ & $100 \%$ \\
\hline
\end{tabular}




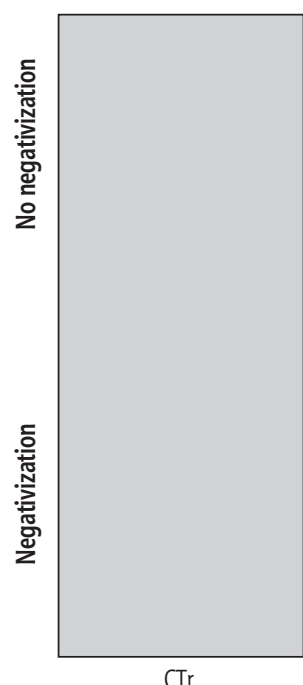

$\mathrm{CTr}$

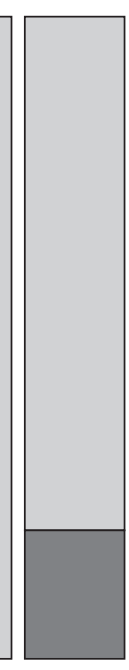

NRS

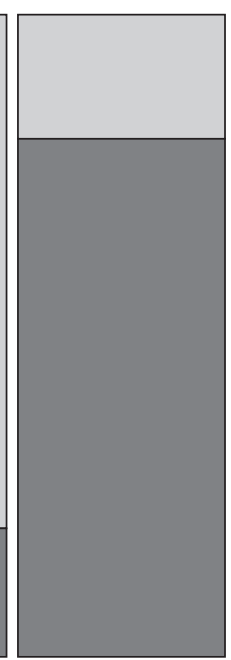

RS

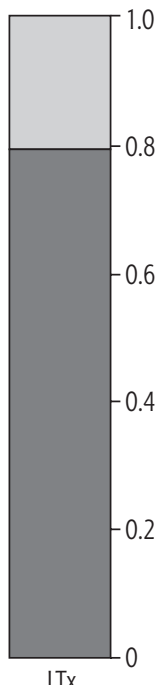

LTx

Fig. 1. Percentage of patients with negative result of Echinococcus multilocularis ELISA test during the two-year follow-up according to treatment received. $\mathrm{CTr}$ - patients receiving pharmacological treatment; NRS - patients who underwent non-radical surgery; RS - patients who underwent radical surgery; LTx - patients after liver transplantation

one month to one year after the surgical procedure. No change in test results was observed in the group of patients receiving pharmacological treatment (Fig. 1).

In $69.7 \%$ of the analysed patients, the result of the Echinococcus Western Blot IgG assay indicated AE at the time of diagnosis. Negativization of Echinococcus

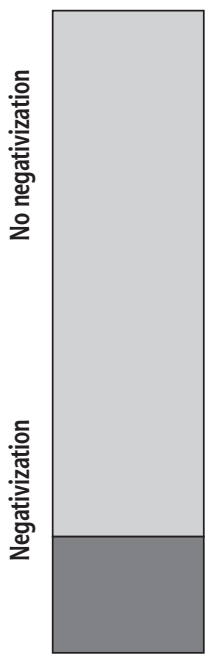

$\mathrm{CTr}$

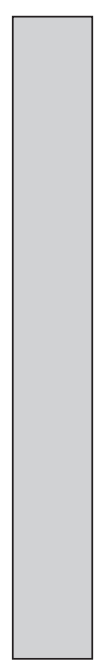

NRS

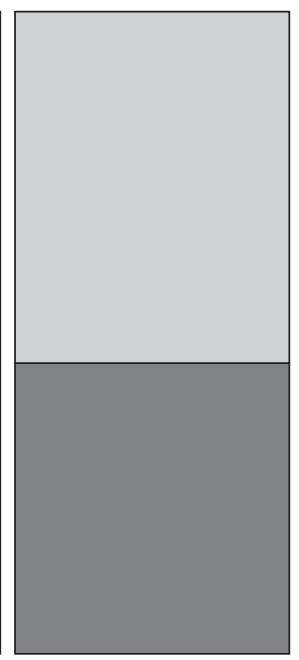

RS

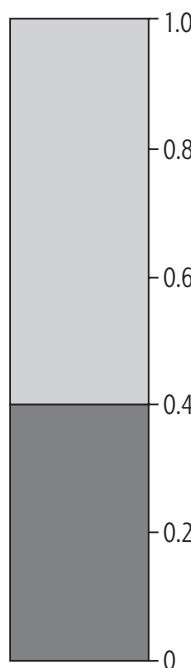

LTX
Fig. 2. Percentage of patients with negative result of Echinococcus Western Blot lgG test during the two-year follow-up according to treatment received. $\mathrm{CTr}$ - patients receiving pharmacological treatment; NRS - patients who underwent non-radical surgery; RS - patients who underwent radical surgery; LTx - patients after liver transplantation

Western Blot IgG (LDBIO Diagnostics) assay results was observed in some patients, among both those who received conservative treatment and those who underwent radical surgery or LTx during the two-year follow-up, but no statistically significant differences (Table 2) were found between treatment groups (Fig. 2).

Table 2. Number of subjects ( $M$ and the percentage values calculated against the sum specified for the given rows (NR) and columns (NC). CTr - patients receiving pharmacological treatment; NRS - patients who underwent non-radical surgery; RS - patients who underwent radical surgery; LTx - patients after liver transplantation. Fisher's exact test for count data was used to examine the independence between the features described in the columns and rows. P-value $=0.1363$

\begin{tabular}{|c|c|c|c|c|}
\hline Patients & & $\begin{array}{l}\text { Echinococcus WB IgG test negative } \\
\text { in the course of two-year treatment }\end{array}$ & $\begin{array}{l}\text { Echinococcus WB IgG test positive } \\
\text { in the course of two-year treatment }\end{array}$ & $\begin{array}{c}\text { All (Echinococcus WB IgG test positive } \\
\text { at the time of diagnosis) }\end{array}$ \\
\hline \multirow[t]{3}{*}{$\mathrm{CTr}$} & $N$ & 2 & 9 & 11 \\
\hline & NR & $18.2 \%$ & $81.8 \%$ & $100 \%$ \\
\hline & NC & $13.3 \%$ & $28.1 \%$ & $23.4 \%$ \\
\hline \multirow[t]{3}{*}{ NRS } & $N$ & 0 & 6 & 6 \\
\hline & NR & $0 \%$ & $100 \%$ & $100 \%$ \\
\hline & $N C$ & $0 \%$ & $18.75 \%$ & $12.8 \%$ \\
\hline \multirow[t]{3}{*}{ RS } & $N$ & 9 & 11 & 20 \\
\hline & NR & $45 \%$ & $55 \%$ & $100 \%$ \\
\hline & NC & $60 \%$ & $34.4 \%$ & $42.6 \%$ \\
\hline \multirow[t]{3}{*}{ LTx } & $N$ & 4 & 6 & 10 \\
\hline & NR & $40 \%$ & $60 \%$ & $100 \%$ \\
\hline & NC & $26.7 \%$ & $18.75 \%$ & $21.3 \%$ \\
\hline \multirow[t]{3}{*}{ Total } & $N$ & 15 & 32 & 47 \\
\hline & NR & $31.9 \%$ & $68.1 \%$ & $100 \%$ \\
\hline & NC & $100 \%$ & $100 \%$ & $100 \%$ \\
\hline
\end{tabular}


Among serum samples from 11 patients after radical surgery or LTx in whom Echinococcus Western Blot IgG assay remained positive, despite negative results of the Echinococcus multilocularis ELISA test, that were additionally examined using Anti-Echinococcus EUROLINE-WB (IgG), negative results were obtained in 6 samples (54.5\%). In 2 samples (18.2\%) test results were borderline, in 2 samples the results were still positive for $\mathrm{AE}$ infection, and in one sample the positive test result did not allow for differentiation between $\mathrm{CE}$ and $\mathrm{AE}$.

\section{Discussion}

The results of serological tests, Echinococcus Western Blot IgG and Echinococcus multilocularis ELISA, were analysed in patients with probable and certain diagnosis of AE. The Echinococcus Western Blot IgG assay is highly sensitive and can differentiate between $\mathrm{CE}$ and $\mathrm{AE}$ in $76 \%$ of cases [8]. In our group of patients, the test indicated $\mathrm{AE}$ at the time of diagnosis in $69.7 \%$ of cases. The assay does not allow for determining whether the parasitic infection is active or not [8], although it may be helpful in monitoring the treatment of patients with $\mathrm{AE}$ [9]. In the group of patients who underwent radical surgery, a gradual disappearance of 16 and $18 \mathrm{kDa}$ bands (change in the test pattern from P3 to P5) followed by a $7 \mathrm{kDa}$ band (change in the test pattern from P5 to P4) or serological negativization was observed one to four years after surgery [9]. In our study, in the group of patients who underwent radical surgery, negativization of the Echinococcus Western Blot IgG (LDBIO Diagnostics) assay was observed in $45 \%$ of patients during two years after surgery and in all patients up to 9 years after surgery. In the remaining cases, i.e. in those patients without test negativization during the two-year follow-up after the surgery, a change in the test pattern from $\mathrm{P} 3$ to $\mathrm{P} 5$ or P4 interpreted as the disappearance of parasitic lesion activity was observed.

In all patients included in the study, a positive result of the Echinococcus multilocularis ELISA test was obtained at the time of diagnosis. This assay contains the native Em2 antigen isolated from the laminar membrane of the hydatid cyst connected to the recombinant antigen II/3-10 [10] and is characterized by high sensitivity (97\%) and specificity (99\%) for AE [10]. For this reason, it is useful, like the immunoblot test, in differentiation between Echinococcus species [11]. Studies using ELISA with purified native Em2 antigen confirmed that in the group of patients with $\mathrm{AE}$ after radical resection of a hepatic lesion, serological negativization was observed in $67 \%$ of patients within one year of surgery [12]. In our study, we observed statistically significant negativization in the group of patients who underwent radical surgery and LTx. It occurred one month to one year after surgery. In patients who underwent non-radical surgery, negativization of the test occurred later. The Echinococcus multilocularis ELISA test was used for assessment of the effectiveness of pharmacological treatment $[13,14]$. However, researchers from various centres report long-term maintenance of positive test results in patients with inactive (calcified) lesions. Our study confirms this thesis, because in the group of patients who did not undergo surgical treatment, no case of negativization of the Echinococcus multilocularis ELISA test was observed during long-term follow-up, with the longest follow-up period being 14 years. This fact is most likely related to the laminar membrane antigens that stimulate the immune response. This is supported by serological negativization observed after removal of AE lesions from the liver $[12,13]$.

The recombinant antigen Em18 (rEm18) is currently considered the most useful in the differentiation between $\mathrm{AE}$ and $\mathrm{CE}[11,15-17]$ as well as in the assessment of the activity of parasitic lesions $[18,19]$. The use of $\mathrm{rEm} 18$ increases the specificity of the test for $\mathrm{AE}$ [15], and thus allows for better differentiation between species compared with the Echinococcus multilocularis ELISA test [11]. The Anti-Echinococcus EUROLINE-WB (IgG) assay combines electrophoretically separated native antigens of vesicle fluid of $E$. multilocularis with recombinant, purified antigens specific for E. multilocularis (Em18, Em95) and E. granulosus (EgAgB). According to the manufacturer's data, the Anti-Echinococcus EUROLINE-WB (IgG) assay has a rate of differentiation between $\mathrm{CE}$ and $\mathrm{AE}$ of $81 \%$.

The use of the Anti-Echinococcus EUROLINE-WB (IgG) assay in the analysed group of 11 patients who underwent surgical treatment or LTx, in whom persistent positive results of the Echinococcus Western blot IgG test were observed despite the negativization of the Em2plus ELISA test, showed that $54.5 \%$ of cases were negative and $18.2 \%$ borderline. These results suggest that this assay is more "dynamic" and becomes negative earlier during effective treatment compared with the Echinococcus Western Blot IgG assay. This hypothesis requires further research on a larger group of patients, including patients treated pharmacologically.

In practice, it can be important in monitoring the activity of parasitic disease and making decisions about termination of pharmacological treatment. On the other hand, there are still patients in everyday practice undergoing surgical treatment due to a focal lesion in the liver, who appear at centres dealing with the treatment 
of echinococcosis late, even one year after surgery. In some of them, when after radical treatment Echinococcus multilocularis ELISA and Anti-Echinococcus EUROLINE-WB (IgG) assays are negative, the Echinococcus Western Blot IgG test may prove useful in establishing further management of patients with suspected $\mathrm{AE}$, including the implementation of antiparasitic treatment and continuation of follow-up of the patient. When interpreting the results of serological tests, the possibility of cross-reactions should be additionally considered. In the case of the Anti-Echinococcus EUROLINE-WB (IgG) assay, false-positive results may occur in other parasitic infections such as Ascaris lumbricoides or Anisakis simplex. Cross-reactivity may also occur when performing the Echinococcus Western Blot IgG assay in the group of patients with neurocysticercosis and schistosomiasis $[8,10]$. In contrast, cross-reactivity with the Em2 plus antigen has been observed in fluke infections (fasciolosis, schistosomiasis) and cystic echinococcosis. There is a risk of cross-reactivity of the Echinococcus multilocularis ELISA test in patients with malignancies including liver cancer and abdominal tumours [20].

Patients after LTx, due to the immunosuppressive treatment used, are in a special risk group because of the high risk of recurrence of a parasitic disease. While in patients undergoing radical surgery WHO-IWGE recommends treatment with benzimidazoles for 2 years after the surgery, in the LTx group there are no clear recommendations [3]. In the analysed group of LTx patients, one patient had recurrence of a parasitic disease (confirmed in a histopathological examination). It was a patient who had himself stopped taking albendazole in the first year after LTx. The recurrence was preceded by positivization of the Echinococcus multilocularis ELISA test and a change in the Echinococcus Western Blot IgG test pattern - a change from $\mathrm{P} 4$ to P3. In the second case, recurrence of AE was suspected based on imaging examinations performed a year after LTx, and positive results of blood serology using Echinococcus multilocularis ELISA and Echinococcus Western Blot IgG assays have been consistently observed since LTx, despite the antiparasitic treatment.

The paper emphasizes the necessity of conducting serological diagnostics of $\mathrm{AE}$ using simultaneously several assays with different levels of sensitivity and specificity in relation to Echinococcus antigens [15]. Interpretation of test results should always take place in conjunction with the results of imaging studies and data on the clinical course of $\mathrm{AE}$, including previous surgical treatment. There is a need to raise awareness among physicians about AE. Invasive diagnostic methods, often performed without previously initiated anti- parasitic treatment, carry the risk of spreading the infection. Performing serological assays in the first place allows this error to be avoided.

\section{Conclusions}

Echinococcus multilocularis ELISA (Bordier Affinity Products) proved to be useful in assessing the activity of $\mathrm{AE}$ in a group of patients who underwent radical surgery or LTx. The negativization of the Echinococcus Western Blot IgG (LDBIO Diagnostics) assay during the two-year follow-up was not statistically significantly related to the type of treatment used.

The results of our study suggest that Anti-Echinococcus EUROLINE-WB (IgG) is a more dynamic test, which at the time of disappearance of AE activity becomes negative earlier - in $54.5 \%$ of cases it was negative when the results of the Echinococcus Western Blot IgG assay were still positive and presented the $\mathrm{P} 4$ or $\mathrm{P} 5$ pattern (did not distinguish between $\mathrm{AE}$ and $\mathrm{CE}$ ).

\section{Disclosure}

The authors report no conflict of interest.

\section{References}

1. Nahorski WL, Knap JP, Pawłowski ZS, et al. Human alveolar echinococcosis in Poland: 1990-2011. PLoS Negl Trop Dis 2013; 7: e1986.

2. McManus DP, Zhang W, Li J, Bartley PB. Echinococcosis. Lancet 2003; 362: 1295-1304.

3. Brunetti E, Kern P, Vuitton DA. Writing Panel for the WHOIWGE. Expert consensus for the diagnosis and treatment of cystic and alveolar echinococcosis in humans. Acta Tropica 2010; 114: 1-16.

4. Graeter T, Kratzer W, Oeztuerk S, et al. Proposal of a computed tomography classification for hepatic alveolar echinococcosis. World J Gastroenterol 2016; 22: 3621-3631.

5. Coskun A, Oztürk M, Karahan OI, et al. Alveolar echinococcosis of the liver: correlative color Doppler US, CT, and MRI study. Acta Radiol 2004; 45: 492-498.

6. Bresson-Hadni S, Laplante JJ, Lenys D, et al. Seroepidemiologic screening of Echinococcus multilocularis infection in a European area endemic for alveolar echinococcosis. Am J Trop Med Hyg 1994; 51: 837-846.

7. Sato N, Namieno T, Furuya K, et al. Contribution of mass screening system to resectability of hepatic lesions involving Echinococcus multilocularis. J Gastroenterol 1997; 32: 351-354.

8. Liance M, Janin V, Breson-Hadni S, et al. Immunodiagnosis of Echinococcus infections; confirmatory testing and species differentiation by a new commercial western blot. J Clin Microbiol 2000; 38: 3718-3721.

9. Tappe D, Grüner B, Kern P, Frosch M. Evaluation of a commercial echinococcus Western Blot assay for serological follow-up of patients with alveolar echinococcosis. Clin Vaccine Immunol 2008; 15: 1633-1637.

10. Gottstein B, Jacquier P, Bresson-Hadni S, et al. Improved primary immunodiagnosis of alveolar echinococcosis in humans 
by an enzyme-linked immunosorbent assay using the Em2plus antigen. J Clin Microbiol 1993; 31: 373-376.

11. Ito A, Ma L, Paul M, et al. Evaluation of Em18-, Em16-, antigen B-western blots, Em2plus ELISA and four other tests for differential serodiagnosis of alveolar and cystic echinococcosis patients in Poland. Int J Parasitol 1998; 47: 95-99.

12. Gottstein B, Tschudi K, Eckert J, Ammann R. Em2-ELISA for the follow-up of alveolar echinococcosis after complete surgical resection of liver lesions. Trans R Soc Trop Med Hyg 1989; 83: 389-393.

13. Ammann RW, Renner EC, Gottstein B, et al. Immunosurveillance of alveolar echinococcosis by specific humoral and cellular tests: long term analysis of the Swiss chemotherapy trial (1976-2001). J Hepatol 2004; 4: 551-559.

14. Ma L, Ito A, Liu YH, et al. Alveolar echinococcosis: Em2plusELISATM and Em 18-Western blots for follow-up after treatment with albendazole. Trans R Soc Trop Med Hyg 1997; 91: 276-478.

15. Knapp J, Sako Y, Grenouillet F, et al. Comparison of the serological tests ICT and ELISA for the diagnosis of alveolar echinococcosis in France. Parasite 2014; 21: 34.

16. Ito A, Xiao N, Liance $M$, et al. Evaluation of an enzyme-linked immunosorbent assay (ELISA) with affinity-purified Em18 and an ELISA with recombinant Em18 for differential diagnosis of alveolar echinococcosis; results of a blind test. J Clin Microbiol 2002; 40: 4161-4165.

17. Ito A, Sako Y, Yamasaki H, et al. Development of Em18-immunoblot and Em18-ELISA for specific diagnosis of alveolar echinococcosis. Acta Trop 2003; 85: 173-182.

18. Ishikawa Y, Sako Y, Itoh S, et al. Serological monitoring of progression of alveolar echinococcosis with multiorgan involvement by use of recombinant Em18. J Clin Microbiol 2009; 47: 3191-3196.

19. Fujimoto $Y$, Ito A, Ishikawa $Y$, et al. Usefulness of recombinant Em18-ELISA to evaluate efficacy of treatment in patients with alveolar echinococcosis. J Gastroenterol 2005; 40: 426-431.

20. Paul M, Stefaniak J, Rychlicki W, et al. Evaluation of a risk of a cross-reactivity with Echinococcus multilocularis-specific Em2plus antigen in patients with liver cancer. Wiad Parazytol 2004; 50: 439-445. 